\title{
The Phase of Ongoing EEG Oscillations Uncovers the Fine Temporal Structure of Conscious Perception
}

\author{
Valentin Wyart ${ }^{1,2,3}$ and Claire Sergent ${ }^{1,2,3}$ \\ ${ }^{1}$ Université Pierre et Marie Curie-Paris 06, Unité Mixte de Recherche (UMR) 7225, S-975, Centre de Recherche de l'Institut Cerveau-Moelle (CRICM), \\ ${ }^{2}$ CNRS (Centre National de la Recherche Scientifique), UMR 7225, CRICM, and ${ }^{3}$ Inserm (Institut National de la Santé et de la Recherche Médicale), \\ UMR S-975, CRICM, Paris F-75013, France \\ Review of Mathewson et al. and Busch et al.
}

Threshold stimulation has proven to be an ideal method for studying conscious perception: across repeated presentations of the same visual stimulus, our perception vividly fluctuates between times when we clearly see the stimulus and times when we do not see it at all. However, the origin of these perceptual fluctuations has been puzzling psychologists for more than a century (Fechner, 1860). Recently, neurophysiologists have related perceptual fluctuations to the large trial-to-trial variability in brain signals, visible from the single-cell level to the scalp electroencephalogram (EEG). This neuronal variability shows up not only in the trial-to-trial fluctuations of evoked sensory responses to near-threshold stimuli, but also in the spontaneous fluctuations of ongoing brain activity (Arieli et al., 1996).

While the search for the neuronal correlates of consciousness has focused on which components of the evoked response correlate with conscious perception (Dehaene et al., 2001), recent results have shown

Received July 15, 2009; revised Aug. 27, 2009; accepted Aug. 27, 2009.

This work was supported by grants from Région Ile-de-France to V.W. and Agence Nationale de la Recherche to C.S. We wish to thank C. Aissani, K.

$\mathrm{N}^{\prime}$ Diaye, and C. Tallon-Baudry for helpful comments on an earlier version of this manuscript.

Correspondence should be addressed to Valentin Wyart, Centre de Recherche de I'Institut Cerveau-Moelle, Bâtiment Médecine Physique et Réadaptation, LENA, 47 boulevard de I'Hôpital, Paris F-75013, France. E-mail: valentin.wyart@upmc.fr.

DOI:10.1523/JNEUROSCI.3410-09.2009

Copyright $\odot 2009$ Society for Neuroscience $\quad$ 0270-6474/09/2912839-03\$15.00/0 that the amplitude of prestimulus ongoing brain signals could predict the perception of upcoming stimuli (Hesselmann et al., 2008). Two recent EEG studies by Mathewson et al. (2009) and Busch et al. (2009) published in The Journal of Neuroscience go one step further by providing a functional link between the fine temporal structure of ongoing brain activity and conscious perception.

Oscillations are one of the most remarkable properties of ongoing electrophysiological signals. During rest, the scalp EEG is dominated by posterior $\sim 10$ $\mathrm{Hz}$ oscillations whose amplitude is modulated by top-down attention (Thut et al., 2006) and correlates with the conscious perception of upcoming near-threshold visual stimuli (van Dijk et al., 2008). These amplitude effects are typically observed over hundreds of milliseconds, i.e., multiple oscillatory cycles. But is the fine temporal structure of these oscillations within a single oscillatory cycle (i.e., the temporal succession of a peak and a trough) related to conscious perception? To examine this issue, Mathewson et al. (2009) and Busch et al. (2009) tested whether the phase of ongoing EEG oscillations could influence the detection of a near-threshold visual stimulus. Using different paradigms, the two studies found that the phase of ongoing $5-10 \mathrm{~Hz}$ oscillations just before stimulus onset correlated with detection.

In both studies, the near-threshold target stimuli were presented for a very short period [12 ms for Mathewson et al. (2009), $6 \mathrm{~ms}$ for Busch et al. (2009)], much shorter than a full oscillatory cycle at $10 \mathrm{~Hz}$. On each trial, the target thus fell into a precise phase for slow oscillations $(<10 \mathrm{~Hz})$. Longer presentation times could have blurred potential phase effects, since the phase would vary significantly between target onset and offset. The analysis of event-related potentials (ERPs) following target onset showed previously reported differences between detected and missed targets, in particular a late positive ERP component peaking at $\sim 400 \mathrm{~ms}$ at anterior electrodes exclusively for consciously detected targets (Sergent et al., 2005).

The two studies then focused on the prestimulus period and looked for amplitude effects in the frequency domain. Both found that the amplitude of prestimulus $\sim 10 \mathrm{~Hz}$ oscillations correlated with the detection of the upcoming target: the smaller the amplitude, the more likely the target would be detected. In particular, Busch et al. (2009) used an exhaustive approach by searching a large time-frequency domain for amplitude effects ( 4 to 50 $\mathrm{Hz},-800$ to $+800 \mathrm{~ms}$ relative to target onset), along with statistical corrections for multiple comparisons. This analysis showed that the observed amplitude effect was centered at $8 \mathrm{~Hz}$, sustained over at least $300 \mathrm{~ms}$, and widely distributed across electrodes [Busch et al. (2009), their Fig. $2 A]$. Besides, this effect accounted for a 
difference of $12 \%$ in detection performance in Busch et al.'s study.

The two studies then looked for phase effects. Whereas Mathewson et al. (2009) sorted their data according to the oscillatory phase at a predefined time-frequency point (10 Hz at target onset) at a single parietal electrode, Busch et al. (2009) again searched a large time-frequency domain based on a phase bifurcation index quantifying the difference in phase distributions between detected and missed targets, computed at each electrode, and followed by appropriate statistical corrections. Both studies found that the phase of ongoing $\sim 10 \mathrm{~Hz}$ oscillations correlated with conscious perception. Busch et al. further showed that this phase effect peaked at $7 \mathrm{~Hz}$ and $-120 \mathrm{~ms}$ relative to target onset at frontal electrodes [(2009), their Fig. $2 B$ ], accounting for a difference of $16 \%$ in detection performance, i.e., slightly more than the amplitude effect. Despite an apparent discrepancy between phase circular histograms [Mathewson et al. (2009), their Fig. 4D; Busch et al. (2009), their Fig. 3B] likely to result from different phase definitions in the two studies, the reported effects coincide in the sense that detected and missed targets were phase locked at opposite phase angles. Peaks and troughs at target onset were associated with lower and higher probabilities of detecting the target, respectively [Mathewson et al. (2009), their Fig. $4 F]$.

Although Busch et al. (2009) assumed that phase and amplitude effects were largely independent, Mathewson et al. (2009) directly assessed their relationship by testing the strength of the phase effect separately for low and high amplitudes. This insightful analysis revealed that the phase effect actually depended on the amplitude of oscillations: no phase effect for low amplitudes, but a significant phase effect for high amplitudes [Mathewson et al. (2009), their Fig. 4B]. This result challenges the assumption that phase and amplitude effects are unrelated. Instead, it advocates in favor of a common perceptual process underlying sensory selection (Schroeder and Lakatos, 2009).

Despite the large convergence between the results of the two studies, there were critical differences between them in terms of experimental design. First, while Mathewson et al. (2009) used a metacontrast mask, i.e., a high-contrast annulus appearing $50 \mathrm{~ms}$ after target offset around the target location to render the target barely detectable, Busch et al. (2009) lowered the target luminance us- ing a psychophysical staircase to determine the threshold for which the target would be consciously detected half of the time. These two distinct approaches draw different constraints on visual perception: backward masking disrupts the detection of high-contrast targets by the interference between target and mask processing, whereas the detection of unmasked lowcontrast targets depends critically on the strength of target processing. This difference could potentially explain why early visual ERPs $(<150 \mathrm{~ms})$ correlate with conscious perception only in Mathewson et al.'s study [(2009), their Fig. 5]; i.e., before mask processing interrupts the visual response to an otherwise supra-threshold target. Second, Busch et al. (2009) made sure that the phase of oscillations would be uniformly distributed across trials at target onset by using a variable temporal delay between fixation onset and target onset (1-2 s), and running a posteriori statistical tests. In contrast, Mathewson et al. (2009) used a fixed temporal delay that could have introduced undesirable phase locking between the ERP to the fixation cross and the phase of $\sim 10 \mathrm{~Hz}$ oscillations at target onset. However, no phase locking is apparent on the ERP at target onset [Mathewson et al. (2009), their Fig. 4F], suggesting that the phase of ongoing $\sim 10$ $\mathrm{Hz}$ oscillations could not be voluntarily controlled by temporal attention to enhance visual processing precisely at target onset.

Together, the results reported in the two studies suggest that perception may rely on an oscillating process, periodically alternating at $5-10 \mathrm{~Hz}$ between excitable and inhibited brain states for which the same near-threshold stimulus is more or less likely to be consciously detected. As noted by Busch et al. (2009), these findings support the proposed role of phase coding in perception. In a neuronal population whose excitability oscillates over time, the strength of the stimulus feature encoded by the population is directly transformed into a phase value: the stronger the stimulus feature, the earlier the phase within each oscillatory cycle (Fries et al., 2007). For instance, such phase coding at $4-8 \mathrm{~Hz}$ has recently been shown to strongly enhance the information carried by spike trains in monkey auditory cortex (Kayser et al., 2009). More generally, flexible interactions between distant neuronal populations, which are the cornerstone of current theories of consciousness, have been proposed to rely on similar phase relations both within (Womelsdorf et al.,
2007) and between frequency bands (Canolty et al., 2006).

Several questions remain to be addressed by future studies. First, does the phase effect depend on top-down attention? Indeed, ongoing $\sim 10 \mathrm{~Hz}$ oscillations are known to be suppressed at the focus of spatial attention (Thut et al., 2006), and the phase effect reported by Mathewson et al. (2009) was only observed for highamplitude oscillations, i.e., in the absence of a strong attentional focus. To determine whether the periodic sampling of sensory information directly reflects the operating regime of spatial attention (VanRullen et al., 2007), one could use a predictive spatial cue to orient attention toward or away from the near-threshold target (Wyart and Tallon-Baudry, 2008), and compare the strength of the phase effect at and away from the focus of spatial attention. Second, is the phase effect specific to visual perception or does it reflect a general perceptual process (Schroeder and Lakatos, 2009)? Given its frontal topography, the phase effect may originate in non-sensory associative regions, but source reconstruction using more EEG channels will be required to conclude [Mathewson et al. (2009) and Busch et al. (2009) used 21 and 32 channels, respectively]. Together, these intriguing results support the view that the fine temporal structure of ongoing brain oscillations dynamically shape our conscious experience (Buzsaki, 2006), and provide a simple yet powerful mechanistic account for the fluctuations of perception across repeated presentation of the same stimulus.

\section{References}

Arieli A, Sterkin A, Grinvald A, Aertsen A (1996) Dynamics of ongoing activity: explanation of the large variability in evoked cortical responses. Science 273:1868-1871.

Busch NA, Dubois J, VanRullen R (2009) The phase of ongoing EEG oscillations predicts visual perception. J Neurosci 29:7869-7876.

Buzsaki G (2006) Rhythms of the brain. Oxford: Oxford UP

Canolty RT, Edwards E, Dalal SS, Soltani M, Nagarajan SS, Kirsch HE, Berger MS, Barbaro NM, Knight RT (2006) High gamma power is phase-locked to theta oscillations in human neocortex. Science 313:1626-1628.

Dehaene S, Naccache L, Cohen L, Bihan DL, Mangin JF, Poline JB, Rivière D (2001) Cerebral mechanisms of word masking and unconscious repetition priming. Nat Neurosci 4:752-758.

Fechner GT (1860) Elemente der Psychophysik. Leipzig: Breitkof und Hartel.

Fries P, Nikolić D, Singer W (2007) The gamma cycle. Trends Neurosci 30:309-316.

Hesselmann G, Kell CA, Eger E, Kleinschmidt A (2008) Spontaneous local variations in on- 
going neural activity bias perceptual decisions. Proc Natl Acad Sci USA 105: 10984-10989.

Kayser C, Montemurro MA, Logothetis NK, Panzeri S (2009) Spike-phase coding boosts and stabilizes information carried by spatial and temporal spike patterns. Neuron 61: 597-608.

Mathewson KE, Gratton G, Fabiani M, Beck DM, Ro T (2009) To see or not to see: prestimulus alpha phase predicts visual awareness. J Neurosci 29:2725-2732.

Schroeder CE, Lakatos P (2009) Low-frequency neuronal oscillations as instruments of sensory selection. Trends Neurosci 32:9-18.

Sergent C, Baillet S, Dehaene S (2005) Timing of the brain events underlying access to consciousness during the attentional blink. Nat Neurosci 8:1391-1400.

Thut G, Nietzel A, Brandt SA, Pascual-Leone A (2006) Alpha-band electroencephalographic activity over occipital cortex indexes visuospatial attention bias and predicts visual target detection. J Neurosci 26:9494-9502.

van Dijk H, Schoffelen JM, Oostenveld R, Jensen O (2008) Prestimulus oscillatory activity in the alpha band predicts visual discrimination ability. J Neurosci 28:1816-1823.

VanRullen R, Carlson T, Cavanagh P (2007) The blinking spotlight of attention. Proc Natl Acad Sci U S A 104:19204-19209.

Womelsdorf T, Schoffelen JM, Oostenveld R, Singer W, Desimone R, Engel AK, Fries P (2007) Modulation of neuronal interactions through neuronal synchronization. Science 316:1609-1612.

Wyart V, Tallon-Baudry C (2008) Neural dissociation between visual awareness and spatial attention. J Neurosci 28:2667-2679. 